\title{
EXISTENCE OF SOLUTIONS IN QUASI-BANACH SPACES FOR EVOLUTIONARY SOBOLEV TYPE EQUATIONS IN RELATIVELY RADIAL CASE
}

\author{
M. A. Sagadeeva ${ }^{1}$, sam79@74.ru, \\ A. S. Rashid ${ }^{1}$, amargivara@gmail.com. \\ ${ }^{1}$ South Ural State University, Chelyabinsk, Russian Federation.
}

\begin{abstract}
Sobolev-type equations (equations not solved for the highest derivative) probably first appeared in the late nineteenth century. The growing recent interest in Sobolev-type equations motivates us to consider them in quasi-Banach spaces. Specifically, this study aims at understanding non-classical models of mathematical physics in quasi-Banach spaces.

This paper carries over the theory of degenerate strongly continuous semigroups obtained earlier in Banach spaces to quasi-Banach spaces. We prove an analogue of the direct Hille-Yosida-Feller-Miyadera-Phillips theorem. As an application of abstract results, we consider the Showalter-Sidorov problem for modified linear Chen-Gurtin equations in quasi-Sobolev spaces.

Keywords: degenerate strong continuous semigroups; quasi-Banach spaces; HilleIosida-Feller-Miadera-Phillips theorem; modified Chen-Gurtin equation; quasi-Sobolev spaces.
\end{abstract}

\section{Introduction}

Given the space $\mathcal{L}(\mathcal{V})$ of bounded linear operators on a Banach space $\mathcal{V}$, refer to a mapping $V^{\bullet} \in C(\mathbb{R} ; \mathcal{L}(\mathcal{V}))$ as a semigroup of operators whenever for all $s, t \in \mathbb{R}_{+}$we have

$$
V^{s} V^{t}=V^{s+t} \text {. }
$$

Usually a semigroup of operators is identified with its graph $\left\{V^{t}: t \in \mathbb{R}_{+}\right\}$. Say that a semigroup $\left\{V^{t}: t \in \mathbb{R}_{+}\right\}$is of class $C_{0}$ (or is a $C_{0}$-semigroup) whenever it is strongly continuous for $t>0$, i.e. for arbitrary $v \in \mathcal{V}$ we have

$$
\lim _{t \rightarrow 0+} V^{t} v=v .
$$

Constructions of this type arise as semigroups of solving operators of the equation

$$
\dot{v}=A v
$$

on $\mathcal{V}$ with a closed densely defined linear operator $A \in \mathcal{C l}(\mathcal{V})$.

A classical result on the solvability of (2) is the Hille-Yosida-Feller-Miyadera-Phillips theorem [1] (the HYFMF theorem) establishing a bijection between the set of semigroups of solving operators and the set of operators called the generators of these semigroups. The conditions for $A$ to be a generator of a semigroup of solving operators of (1), called [2] the radiality of $A$, amount to certain requirements on the resolvent set $\rho(A)$ and the resolvent $R_{\mu}(A)$ of $A$. The theory of $C_{0}$-semigroups was later extended to Fréchet spaces [3, Ch. 9]. 
A semigroup $\left\{V^{t}: t \in \mathbb{R}\right\}$ is called degenerate whenever its identity element

$$
P=\mathrm{s}-\lim _{t \rightarrow 0+} V^{t}
$$

is a projection in $\mathcal{V}$. Degenerate $C_{0}$-semigroups of operators appeared for the first time in [4] as semigroups of solving operators of linear evolutionary Sobolev-type equations

$$
L \dot{u}=M u,
$$

where $\mathfrak{U}$ and $\mathfrak{F}$ are Banach spaces, $L \in \mathcal{L}(\mathfrak{U} ; \mathfrak{F})$ is a bounded linear operator, and $M \in \mathcal{C l}(\mathfrak{U} ; \mathfrak{F})$ is a closed and densely defined linear operator. The complete theory of these semigroups is presented in [5, Ch. 2] and extended to Fréchet spaces in [6].

The equations of the form (3) appeared for the first time at the end of 19th century in Poincaré's works, and in the second half of the 20th century Sobolev began their systematic study (see [7] for a great survey). Since the interest in Sobolev-type equations is growing fast recently (see [8] - [11] for instance), it becomes necessary to consider them in quasiBanach spaces. This is forced not as much by the desire to expand the theory as by the intention to interpret the nonclassical models of mathematical physics [12] in quasi-Banach spaces [13].

The Sobolev-type equations (2) are called dynamical when their solutions extend to the whole of $\mathbb{R}$ and evolutionary when their solutions exist only on $\mathbb{R}_{+}$[14]. The existence of solutions for the dynamical Sobolev-type equations is proved in article [15].

Aside from the introduction and bibliography, this article consists of three sections. In the auxiliary first section we consider quasi-Banach spaces and closed bounded linear operators on them. We also introduce quasi-Sobolev spaces and construct in them the powers of the Laplace quasi-operator. The second section we find conditions on the operators $L$ and $M$ leading to strongly continuous degenerate semigroups of operators in quasi-Banach spaces $\mathfrak{U}$ and $\mathfrak{F}$. In other words, we prove the direct assertion of the generalization of the HYFMF theorem to quasi-Banach spaces. In the last section we consider evolutionary Sobolev-type equations with a relatively radial operator in quasiBanach spaces and, as an example, mention the "quasi-Banach" analog of the homogeneous Dirichlet problem in a bounded region with smooth boundary for the modified linear ChenGurtin equation (see [5], [11], [16] for instance)

$$
(\lambda-\Delta) u_{t}=\beta \Delta u-\alpha \Delta^{2} u+f
$$

with the Showalter-Sidorov initial condition [17]. The bibliography, not intended to be complete, reflects the authors' tastes and preferences.

\section{Closed Linear Operators in Quasi-Sobolev Spaces}

Quasi-Banach spaces are metrizable complete quasi-normed spaces. The spaces $\ell_{q}$ of sequences for $q \in(0,1)$ are well-known examples of quasi-Banach spaces (for $q \in[1,+\infty$ ) they are Banach spaces). Henceforth denote by $\left\{\lambda_{k}\right\} \subset \mathbb{R}_{+}$a monotone sequence with

$$
\lim _{k \rightarrow \infty} \lambda_{k}=+\infty \text {. }
$$

Refer as a quasi-Sobolev space to the quasi-Banach space

$$
\ell_{q}^{m}=\left\{u=\left\{u_{k}\right\}: \sum_{k=1}^{\infty}\left(\lambda_{k}^{\frac{m}{2}}\left|u_{k}\right|\right)^{q}<+\infty\right\}
$$


equipped with the quasi-norm

$$
{ }_{q}^{m}\|u\|=\left(\sum_{k=1}^{\infty}\left(\lambda_{k}^{\frac{m}{2}}\left|u_{k}\right|\right)^{q}\right)^{1 / q}, m \in \mathbb{R} .
$$

It is obvious that $\ell_{q}^{m}$ is a Banach space for $q \in[1,+\infty)$ and $\ell_{q}^{0}=\ell_{q}$; moreover, we have continuous dense embeddings $\ell_{q}^{n} \hookrightarrow \ell_{q}^{m}$ for $n \geq m$ and $q \in \mathbb{R}_{+}$.

Given two quasi-Banach spaces $\mathfrak{U}$ and $\mathfrak{F}$, a linear operator $L: \mathfrak{U} \rightarrow \mathfrak{F}$ is called continuous whenever

$$
\lim _{k \rightarrow \infty} L u_{k}=L\left(\lim _{k \rightarrow \infty} u_{k}\right)
$$

for every sequence $\left\{u_{k}\right\} \subset \mathfrak{U}$ converging in $\mathfrak{U}$. It is not difficult to show that a linear operator $L: \mathfrak{U} \rightarrow \mathfrak{F}$ is continuous precisely when it is bounded (that is, maps bounded sets into bounded sets). The linear space $\mathcal{L}(\mathfrak{U} ; \mathfrak{F})$ of bounded linear operators equipped with the quasi-norm

$$
\mathcal{L}(\mathfrak{L} ; \mathfrak{F})\|L\|=\sup _{\mathfrak{U}\|u\|=1} \mathfrak{F}\|L u\|,
$$

where $\mathfrak{U}\|\cdot\|$ and $\mathfrak{F}\|\cdot\|$ are quasi-norms in $\mathfrak{U}$ and $\mathfrak{F}$, is a quasi-Banach space. A sequence $\left\{L_{k}\right\} \subset \mathcal{L}(\mathfrak{U} ; \mathfrak{F})$ is called strongly converging to $L \in \mathcal{L}(\mathfrak{U} ; \mathfrak{F})$ whenever $\mathfrak{F}\left\|L_{k} u-L u\right\| \rightarrow 0$ as $k \rightarrow \infty$ for every $u \in \mathfrak{U}$ and uniformly converging whenever $\mathcal{L}(\mathfrak{U} ; \mathfrak{F})\left\|L_{k}-L\right\| \rightarrow 0$ as $k \rightarrow \infty$.

Theorem 1. (Analog of the Banach-Steinhaus theorem) A sequence $\left\{L_{k}\right\} \subset \mathcal{L}(\mathfrak{U} ; \mathfrak{F})$ uniformly converges to an operator $L \in \mathcal{L}(\mathfrak{U} ; \mathfrak{F})$ on some dense linear space $\mathfrak{U}$ of $\mathfrak{U}$ precisely when

(i) the sequence $\left\{L_{k}\right\}$ is bounded;

(ii) the sequence $\left\{L_{k}\right\}$ strongly converges to $L$ on $\stackrel{\circ}{\mathfrak{U}}$.

A linear operator $L: \mathfrak{U} \rightarrow \mathfrak{F}$ is called closed whenever its graph

$$
\operatorname{graph} L=\{(u, f) \in \mathfrak{U} \times \mathfrak{F}: f=L u\}
$$

is closed in the quasi-norm graphL $\|u\|=_{\mathfrak{U}}\|u\|+_{\mathfrak{F}}\|L u\|$.

Theorem 2. If $L \in \mathcal{L}(\mathfrak{U} ; \mathfrak{F})$ then $L$ is a closed operator.

Theorem 3. If $L: \mathfrak{U} \rightarrow \mathfrak{F}$ is a closed linear operator with the domain $\operatorname{dom} L=\mathfrak{U}$ then $L \in \mathcal{L}(\mathfrak{U} ; \mathfrak{F})$.

Theorem 4. If $L: \mathfrak{U} \rightarrow \mathfrak{F}$ is a closed operator and $L^{-1}: \mathfrak{F} \rightarrow \mathfrak{U}$ exists then $L^{-1}$ is a closed operator.

A linear operator $L: \mathfrak{U} \rightarrow \mathfrak{F}$ is called densely defined whenever the closure of the linear space $\overline{\operatorname{dom} L}=\mathfrak{U}$. Denote the linear space of closed densely defined operators by $\mathcal{C l}(\mathfrak{U} ; \mathfrak{F})$.

Example 1. Taking $\mathfrak{U}=\ell_{q}^{m+2}$ and $\mathfrak{F}=\ell_{q}^{m}$, consider the operator $\Lambda^{n} u=\left\{\lambda_{k}^{n} u_{k}\right\}$ with $n \in \mathbb{N}$, where $\left\{u_{k}\right\} \subset \mathfrak{U}$, and a monotone sequence $\left\{\lambda_{k}\right\} \subset \mathbb{R}_{+}$with

$$
\lim _{k \rightarrow \infty} \lambda_{k}=+\infty
$$


It is not difficult to see that $\Lambda^{n} \in \mathcal{C l}(\mathfrak{U} ; \mathfrak{F})$ with $\operatorname{dom} \Lambda^{n}=\ell_{q}^{m+2 n}$; furthermore, $\Lambda^{n}: \ell_{q}^{m+2 n} \rightarrow \ell_{q}^{m}$ is a top-linear isomorphism.

Theorem 5. If an operator $\widetilde{L}: \mathfrak{U} \rightarrow \mathfrak{F}$ is densely defined and its quasi-norm satisfies

$$
\|\widetilde{L}\|=\sup _{u \in \operatorname{dom} M \backslash\{0\}} \frac{\mathfrak{F}\|\widetilde{L} u\|}{\mathfrak{U}\|u\|}<+\infty,
$$

then it extends uniquely to an operator $L \in \mathcal{L}(\mathfrak{U} ; \mathfrak{F}) ;$ moreover, $\mathcal{L}(\mathfrak{U} ; \mathfrak{F})\|L\|=\|\widetilde{L}\|$.

Proofs of Theorems 1-5 are similar to the case of Banach spaces, and so we omit them.

\section{Degenerate $C_{0}$-Semigroups of Operators}

Consider two quasi-Banach spaces $\mathfrak{U}$ and $\mathfrak{F}$, as well as two operators $L \in \mathcal{L}(\mathfrak{U} ; \mathfrak{F})$ and $M \in \mathcal{C l}(\mathfrak{U} ; \mathfrak{F})$. Following [2], [4], and [5], introduce the L-resolvent set

$$
\rho^{L}(M)=\left\{\mu \in \mathbb{C}:(\mu L-M)^{-1} \in \mathcal{L}(\mathfrak{F} ; \mathfrak{U})\right\}
$$

and the $L$-spectrum $\sigma^{L}(M)=\mathbb{C} \backslash \rho^{L}(M)$ of the operator $M$. It is not difficult to see that $\rho^{L}(M)$ is always open, and so the $L$-spectrum of $M$ is always closed (see [15]).

Example 2. Take $\mathfrak{U}=\ell_{q}^{m+2}$ and $\mathfrak{F}=\ell_{q}^{m}$ with $m \in \mathbb{R}$ and $q \in \mathbb{R}_{+}$. Construct the operators $L=\lambda-\Lambda$ and $M=\alpha \Lambda^{2}+\beta \Lambda$ with $\Lambda$ and $\Lambda^{2}$ of Example 1. It is not difficult to show that the $L$-spectrum $\sigma^{L}(M)$ of $M$ consists of the points $\mu_{k}=\left(\alpha \lambda_{k}^{2}+\beta \lambda_{k}\right)\left(\lambda-\lambda_{k}\right)^{-1}$ for $k \in \mathbb{N}$, taking their multiplicities into account.

Definition 1. An operator $M$ is called $p$-radial with respect to an operator $L$ (or, briefly, $(L, p)$-radial) whenever

(i) $\exists a \in \mathbb{R} \forall \mu>a \mu \in \rho^{L}(M)$;

(ii) $\exists K>0 \forall \mu_{k}>a, k=\overline{0, p}, \forall n \in \mathbb{N}$

$$
\max \left\{\mathcal{L}(\mathfrak{U})\left\|\left(R_{(\mu, p)}^{L}(M)\right)^{n}\right\|, \mathcal{L}(\mathfrak{F})\left\|\left(L_{(\mu, p)}^{L}(M)\right)^{n}\right\|\right\} \leq \frac{K}{\prod_{k=0}^{p}\left(\mu_{k}-a\right)^{n}} .
$$

Here

$$
R_{(\mu, p)}^{L}(M)=\prod_{k=0}^{p} R_{\mu_{k}}^{L}(M) \quad \text { and } \quad L_{(\mu, p)}^{L}(M)=\prod_{k=0}^{p} L_{\mu_{k}}^{L}(M)
$$

are the right and left $(L, p)$-resolvents of $M$, while in turn, $R_{\mu}^{L}(M)=(\mu L-M)^{-1} L$ and $L_{\mu}^{L}(M)=L(\mu L-M)^{-1}$ are the right and left $L$-resolvents of $M$.

Example 3. For the spaces $\mathfrak{U}$ and $\mathfrak{F}$ as well as the operators $L$ and $M$ of Example 2 verify that for arbitrary $\lambda, \beta \in \mathbb{R}$ and $\alpha \in \mathbb{R}_{+}$the operator $M$ is $(L, 0)$-radial. Indeed, for all $k \in \mathbb{N}, \lambda_{k} \neq \lambda$, the points $\sigma^{L}(M)$ lie in $\mathbb{R}$; moreover,

$$
\lim _{k \rightarrow \infty} \mu_{k}=-\infty,
$$


which ensures the fulfillment of conditions (i) in Definition 1. Furthermore,

$$
R_{\mu}^{L}(M)= \begin{cases}\sum_{k=1}^{\infty}\left(\mu-\mu_{k}\right)^{-1} e_{k} & \text { if } \lambda_{k} \neq \lambda \text { for all } k \in \mathbb{N} \\ \sum_{k \in \mathbb{N}: k \neq \ell}\left(\mu-\mu_{k}\right)^{-1} e_{k} & \text { if } \lambda_{\ell}=\lambda \text { for some } \ell \in \mathbb{N}\end{cases}
$$

Here $e_{k}=(0, \ldots, 0,1,0, \ldots, 0)$ with 1 in slot $k$. For $a>\max \left\{\mu_{k}\right\}$ the fulfillment of (ii) in Definition 1 is obvious. For the left $L$-resolvent $L_{\mu}^{L}(M)$ of $M$ we verify this condition similarly.

Given the space $\mathcal{L}(\mathcal{V})$ of bounded linear operators on a quasi-Banach space $\mathcal{V}$, a mapping $V^{\bullet} \in C^{\infty}\left(\mathbb{R}_{+} ; \mathcal{L}(\mathcal{V})\right)$ is called a semigroup of operators whenever

$$
V^{s} V^{t}=V^{s+t} \text { for all } s, t \in \mathbb{R}_{+} .
$$

As above, identify a semigroup with its graph $\left\{V^{t}: t \in \mathbb{R}_{+}\right\}$and call it strongly continuous (or a $C_{0}$-semigroup) whenever it is strongly continuous for $t>0$, i.e.

$$
\lim _{t \rightarrow 0+} V^{t} v=v
$$

for every $v \in \mathcal{V}$.

Denote by $\mathfrak{U}^{0}$ and $\mathfrak{F}^{0}$ the kernels $\operatorname{ker} R_{(\mu, p)}^{L}(M)$ and $\operatorname{ker} L_{(\mu, p)}^{L}(M)$, which clearly are linear subspaces. Denote by $\mathfrak{U}^{1}$ and $\mathfrak{F}^{1}$ the closures of $\operatorname{im} R_{(\mu, p)}^{L}(M)$ and $\operatorname{im} L_{(\mu, p)}^{L}(M)$, while by $\tilde{\mathfrak{U}}$ and $\tilde{\mathfrak{F}}$ the closures of the linear spaces $\mathfrak{U}^{0} \dot{+} \operatorname{im} R_{(\mu, p)}^{L}(M)$ and $\mathfrak{F}^{0} \dot{+} \operatorname{im} L_{(\mu, p)}^{L}(M)$ in the quasi-norms of $\mathfrak{U}$ and $\mathfrak{F}$.

Theorem 6. If $M$ is an $(L, p)$-radial operator with $p \in\{0\} \cup \mathbb{N}$ then $L$ and $M$ together generate a semigroup of operators of class $C_{0}$ defined on the subspace $\tilde{\mathfrak{U}}(\tilde{\mathfrak{F}})$.

Remark 1. We can obtain the form of operators in the semigroup using the HilleWidder-Post approximation [18]

$$
\begin{aligned}
U^{t} & =s-\lim _{k \rightarrow \infty}\left(\left(L-\frac{t}{k} M\right)^{-1} L\right)^{k}=s-\lim _{k \rightarrow \infty}\left(\frac{k}{t} R_{\frac{k}{t}}^{L}(M)\right)^{k}, \\
F^{t} & =s-\lim _{k \rightarrow \infty}\left(L\left(L-\frac{t}{k} M\right)^{-1}\right)^{k}=s-\lim _{k \rightarrow \infty}\left(\frac{k}{t} L_{\frac{k}{t}}^{L}(M)\right)^{k} .
\end{aligned}
$$

The proof of this theorem is quite bulky, but analogous to the case of Banach spaces (see [4], [5, Ch. 3], and [18] for instance). Therefore, we omit it.

Example 4. Take $\mathfrak{U}, \mathfrak{F}, L$, and $M$ of Example 2. By Example 3, Theorem 6 yields

$$
U^{t}= \begin{cases}\sum_{k=1}^{\infty} e^{\mu_{k} t} e_{k} & \text { if } \lambda_{k} \neq \lambda \text { for all } k \in \mathbb{N}, \\ \sum_{k \in \mathbb{N}: k \neq \ell} e^{\mu_{k} t} e_{k} & \text { if } \lambda_{\ell}=\lambda \text { for some } \ell \in \mathbb{N} .\end{cases}
$$


We can construct a strongly continuous semigroup $\left\{F^{t}: t \in \mathbb{R}_{+}\right\}$similarly.

Refer to a strongly continuous semigroup $\left\{V^{t}: t \in \mathbb{R}_{+}\right\}$as degenerate whenever its identity element

$$
V^{0}=\mathrm{s}^{-} \lim _{t \rightarrow 0+} V^{t}
$$

is a projection in $\mathcal{V}$. Observe in addition that in Definition 1 we can put $a=0$ in view of the change of variables $u(t)=e^{a t} v(t)$ in (2) and notation $M:=M-a L$. Assuming that, put $S_{a, \theta}^{L}(M)=S_{\theta}^{L}(M)$.

Definition 2. An operator $M$ is called strongly $(L, p)$-radial on the right (left) whenever it is $(L, p)$-radial and

$$
\mathfrak{u}\left\|R_{(\mu, p)}^{L}(M)(\lambda L-M)^{-1} M u\right\| \leq \frac{\operatorname{const}(u)}{(\lambda-a) \prod_{k=0}^{p}\left(\mu_{k}-a\right)}, \quad \forall u \in \operatorname{dom} M
$$

(there exists a dense linear space $\stackrel{\circ}{\mathfrak{F}}$ of $\mathfrak{F}$ such that

$$
\left.\mathfrak{F}\left\|M(\lambda L-M)^{-1} L_{(\mu, p)}^{L}(M) f\right\| \leq \frac{\operatorname{const}(f)}{(\lambda-a) \prod_{k=0}^{p}\left(\mu_{k}-a\right)} \quad \forall f \in \stackrel{\circ}{\mathfrak{F}}\right)
$$

for arbitrary $\lambda, \mu_{0}, \mu_{1}, \ldots, \mu_{p}>a$.

Theorem 7. For $p \in\{0\} \cup \mathbb{N}$, if an operator $M$ is strongly $(L, p)$-radial on the right (left) then the strongly continuous semigroup $\left\{U^{t}: t \in \mathbb{R}_{+}\right\}\left(\left\{F^{t}: t \in \mathbb{R}_{+}\right\}\right)$is degenerate.

Proof is similar to the case of Banach spaces, but quite laborious (see [5, Ch. 2] for instance). Therefore, we only sketch it. Firstly, basing on the $(L, p)$-radiality of $M$, we show that the kernel $\operatorname{ker} R_{(\mu, p)}^{L}(M)=\mathfrak{U}^{0}$ and closure of the image $\overline{\operatorname{im} R_{(\mu, p)}^{L}(M)}=\mathfrak{U}^{1}$ are independent of $\mu=\left(\mu_{0}, \mu_{1}, \ldots, \mu_{p}\right)$ with $\mu_{k} \in \rho^{L}(M)$. Then we show that $\mathfrak{U}^{0}=\operatorname{ker} U^{t}$ for all $t \in \mathbb{R}_{+}$and

$$
u=\lim _{t \rightarrow 0+} U^{t} u
$$

for all $u \in \mathfrak{U}^{1}$. Therefore, under the condition that $M$ is strongly $(L, p)$-radial on the right we infer that the projection

$$
P=\mathrm{s}-\lim _{t \rightarrow 0+} U^{t}
$$

exists. Observe also that Theorem 1 is crucial here and at the previous stage. Similarly we establish the existence of the projection

$$
Q=\mathrm{s}^{-} \lim _{t \rightarrow 0+} F^{t}
$$

The main corollary of the strong $(L, p)$-radiality of $M$ on the right (left) is the splitting

$$
\mathfrak{U}=\tilde{\mathfrak{U}}=\mathfrak{U}^{0} \oplus \mathfrak{U}^{1} \quad\left(\mathfrak{F}=\tilde{\mathfrak{F}}=\mathfrak{F}^{0} \oplus \mathfrak{F}^{1}\right),
$$


where $\mathfrak{U}^{0}$ and $\mathfrak{F}^{0}$ are the kernels of the projections $P=\mathrm{s}^{-} \lim _{t \rightarrow 0+} U^{t}$ and $Q=\mathrm{s}-\lim _{t \rightarrow 0+} F^{t}$, while $\mathfrak{U}^{1}$ and $\mathfrak{F}^{1}$ are their images. For $k=0,1$, denote by $L_{k}$ and $M_{k}$ the restrictions of $L$ and $M$ to $\mathfrak{U}^{k}$ and $\operatorname{dom} M \cap \mathfrak{U}^{k}$.

Corollary 1. For $p \in\{0\} \cup \mathbb{N}$, if $M$ is strongly $(L, p)$-radial on both right and left then $L_{k} \in \mathcal{L}\left(\mathfrak{U}^{k} ; \mathfrak{F}^{k}\right)$ and $M_{k} \in \mathcal{C l}\left(\mathfrak{U}^{k} ; \mathfrak{F}^{k}\right)$ for $k=0,1$; furthermore, the operator $M_{0}^{-1} \in \mathcal{L}\left(\mathfrak{F}^{0} ; \mathfrak{U}^{0}\right)$ exists.

Put $H=M_{0}^{-1} L_{0}$ and $G=L_{0} M_{0}^{-1}$. Obviously, $H \in \mathcal{L}\left(\mathfrak{U}^{0}\right)$ and $G \in \mathcal{L}\left(\mathfrak{F}^{0}\right)$.

Corollary 2. Under the assumptions of Corollary 1 , the operators $H$ and $G$ are nilpotent of degree at most $p$.

Definition 3. An operator $M$ is called strongly $(L, p)$-radial whenever it is strongly $(L, p)$ radial on the left and

$$
\mathcal{L}(\mathfrak{F} ; \mathfrak{L})\left\|R_{(\mu, p)}^{L}(M)(\lambda L-M)^{-1}\right\| \leq \frac{K}{(\lambda-a) \prod_{k=0}^{p}\left(\mu_{k}-a\right)}
$$

for arbitrary $\lambda, \mu_{0}, \ldots, \mu_{p}>a$.

Observe that a strongly $(L, p)$-radial operator $M$ is obviously strongly $(L, p)$-radial on the right.

Theorem 8. For $p \in\{0\} \cup \mathbb{N}$, if $M$ is a strongly $(L, p)$-radial operator then the operator $L_{1}^{-1} \in \mathcal{L}\left(\mathfrak{F}^{1} ; \mathfrak{U}^{1}\right)$ exists.

Proof is similar to the case of Banach spaces (see [5, Ch. 2] for instance), and so we omit it.

Construct the operators

$$
\begin{aligned}
& S=L_{1}^{-1} M_{1}: \operatorname{dom} M \cap \mathfrak{U}^{1} \rightarrow \mathfrak{U}^{1}, \\
& T=M_{1} L_{1}^{-1}: M[\operatorname{dom} M] \cap \mathfrak{F}^{1} \rightarrow \mathfrak{F}^{1} .
\end{aligned}
$$

It is not difficult to show that $S \in \mathcal{C l}\left(\mathfrak{U}^{1}\right)$ and $T \in \mathcal{C l}\left(\mathfrak{F}^{1}\right)$.

Corollary 3. Under the assumptions of Theorem 8, the operators $S$ and $T$ are radial.

Remark 2. We emphasize that the strong $(L, p)$-radiality of $M$ implies:

1) the existence of degenerate $C_{0}$-semigroups $\left\{U^{t}: t \in \mathbb{R}_{+}\right\}$and $\left\{F^{t}: t \in \mathbb{R}_{+}\right\}$of (4);

2) the existence of their identity elements, the projections $P \in \mathcal{L}(\mathfrak{U})$ and $Q \in \mathcal{L}(\mathfrak{F})$, thanks to which the quasi-Banach spaces $\mathfrak{U}$ and $\mathfrak{F}$ split in the direct sums (5);

$3)$ the splitting of the actions of the operators $L_{k} \in \mathcal{L}\left(\mathfrak{U}^{k} ; \mathfrak{F}^{k}\right)$ and $M_{k} \in \mathcal{C l}\left(\mathfrak{U}^{k} ; \mathfrak{F}^{k}\right)$ for $k=0,1$ and the existence of the operators $M_{0}^{-1} \in \mathcal{L}\left(\mathfrak{F}^{0} ; \mathfrak{U}^{0}\right)$ and $L_{1}^{-1} \in \mathcal{L}\left(\mathfrak{F}^{1} ; \mathfrak{U}^{1}\right)$;

4) the nilpotency of the operators $H$ and $G$ and the radiality of the operators $S$ and $T$.

We call precisely these statements the generalization of the direct HYFMF theorem to quasi-Banach spaces. 


\section{Evolutionary Sobolev-Type Equations in Quasi-Sobolev Spaces}

Given two quasi-Banach spaces $\mathfrak{U}$ and $\mathfrak{F}$ as well as two operators $L \in \mathcal{L}(\mathfrak{U} ; \mathfrak{F})$ and $M \in \mathcal{C l}(\mathfrak{U} ; \mathfrak{F})$, consider the linear evolutionary Sobolev-type equation

$$
L \dot{u}=M u .
$$

Refer to a vector function $u \in C^{\infty}\left(\mathbb{R}_{+} ; \mathfrak{U}\right)$ satisfying (6) pointwise as a (classical) solution to this equation. Refer to a solution $u=u(t)$ to $(6)$ as a solution to a weak initial value problem (in the sense of Krein) whenever in addition

$$
\lim _{t \rightarrow 0+} u(t)=u_{0}
$$

for some $u_{0} \in \mathfrak{U}$.

Definition 4. A set $\mathfrak{P} \subset \mathfrak{U}$ is called a phase space of (6) whenever

(i) every solution $u=u(t)$ to (6) lies in $\mathfrak{P}$ pointwise, that is, $u(t) \in \mathfrak{P}$ for arbitrary $t \in \mathbb{R}_{+}$;

(ii) for every $u_{0} \in \mathfrak{P}$ there exists a unique solution to the problem (6), (7).

Theorem 9. For $p \in\{0\} \cup \mathbb{N}$, if the operator $M$ is strongly $(L, p)$-radial then the subspace $\mathfrak{U}^{1}$ is the phase space of $(6)$.

Proof.

Firstly, Remark 2 reduces (6) to the equivalent system

$$
H \dot{u}^{0}=u^{0}, \dot{u}^{1}=S u^{1}, \quad u^{1}=P u, u^{0}=u-u^{1} .
$$

Differentiating the first equation with respect to $t$ and multiplying successively on $H$ on the left, we obtain

$$
0=H^{p+1}\left(u^{0}\right)^{(p+1)}=H^{p}\left(u^{0}\right)^{(p)}=\ldots=H \dot{u}^{0}=u^{0} .
$$

Secondly, for the second equation in (8) for arbitrary $u_{0}^{1} \in \mathfrak{U}^{1}$ there exists a unique solution $u^{1}(t)=V^{t} u_{0}^{1}$ to the problem

$$
\lim _{t \rightarrow 0+} u^{1}(t)=u_{0}^{1}
$$

where $V^{t}$ is the semigroup of the form

$$
V^{t}=s-\lim _{k \rightarrow \infty}\left(\left(\mathbb{I}-\frac{t}{k} S\right)^{-1}\right)^{k}=s-\lim _{k \rightarrow \infty}\left(\frac{k}{t} R_{\frac{k}{t}}(S)\right)^{k}, \quad t \in \mathbb{R}_{+} .
$$

Example 5. Take $\mathfrak{U}, \mathfrak{F}, L$, and $M$ as in Example 2. For arbitrary $m, \lambda, \beta \in \mathbb{R}$ and $q, \alpha \in \mathbb{R}_{+}$the phase space of (6) is the subspace

$$
\mathfrak{U}^{1}=\left\{\begin{array}{l}
\mathfrak{U} \text { if } \lambda_{k} \neq \lambda \text { for all } k \in \mathbb{N} \\
\left\{u \in \mathfrak{U}: u_{k}=0, \lambda_{k}=\lambda\right\}
\end{array}\right.
$$

Suppose as above that $\mathfrak{U}$ and $\mathfrak{F}$ are quasi-Banach spaces and take two operators $L \in$ $\mathcal{L}(\mathfrak{U} ; \mathfrak{F})$ and $M \in \mathcal{C} l(\mathfrak{U} ; \mathfrak{F})$ so that $M$ is strongly $(L, p)$-radial with $p \in\{0\} \cup \mathbb{N}$. Consider the weak (in the sense of Krein) Showalter-Sidorov problem

$$
\lim _{t \rightarrow 0+} P\left(u(t)-u_{0}\right)=0
$$


for the linear inhomogeneous evolutionary Sobolev-type equation

$$
L \dot{u}=M u+f
$$

where the vector function $f:[0, \tau] \rightarrow \mathfrak{U}$ with $\tau \in \mathbb{R}_{+}$is defined below. Put $f=f^{0}+f^{1}$, $f^{1}=Q f$ and $f^{0}=f-f^{1}$.

Theorem 10. For $p \in\{0\} \cup \mathbb{N}$, if $M$ is a strongly $(L, p)$-radial operator then, given a vector function $f=f(t)$ with $f^{0} \in C^{p+1}\left((0, \tau) ; \mathfrak{F}^{0}\right)$ and $f^{1} \in C\left((0, \tau) ; \mathfrak{F}^{1}\right)$ as well as a vector $u_{0} \in \mathfrak{U}$, there exists a unique solution $u \in C^{1}((0, \tau) ; \mathfrak{U})$ to problem $(9)$ for equation (10), which in addition is of the form

$$
u(t)=-\sum_{k=0}^{p} H^{k} M_{0}^{-1} f^{0(k)}(t)+U^{t} u_{0}+\int_{0}^{t} U^{t-s} L_{1}^{-1} f^{1}(s) d s .
$$

Indeed, a straightforward verification shows that $u=u(t)$ satisfies (10) and (9). The uniqueness follows from Theorem 10.

As an illustration, consider the modified Chen-Gurtin equation [5],[11],[16]

$$
(\lambda-\Lambda) u_{t}=\left(\alpha \Lambda^{2}+\beta \Lambda\right) u+f, \quad \lambda, \beta \in \mathbb{R}, \alpha \in \mathbb{R}_{+},
$$

in the quasi-Sobolev spaces $\mathfrak{U}=\ell_{q}^{m+2}$ and $\mathfrak{F}=\ell_{q}^{m}$ with $m \in \mathbb{R}$ and $q \in \mathbb{R}_{+}$. Specify the domain $\operatorname{dom}\left(\alpha \Lambda^{2}+\beta \Lambda\right)=\ell_{q}^{m+4}$. Taking the operators $L$ and $M$ as in Example 2, we reduce (11) to the form (10). Example 3 shows that $M$ is an $(L, 0)$-radial operator. It is obvious that the estimates in Definition 2 hold.

In order to pose the Showalter-Sidorov problem, construct the projection $P$. To this end, consider the degenerate $C_{0}$-semigroup constructed in Example 4 . We obtain

$$
P=\text { s- }_{t \rightarrow 0+} U^{t}= \begin{cases}\mathbb{I} & \text { if } \lambda_{k} \neq \lambda \text { for all } k \in \mathbb{N} \\ \mathbb{I}-\sum_{k \in \mathbb{N}: k=\ell} e_{k} & \text { if } \lambda_{\ell}=\lambda \text { for some } \ell \in \mathbb{N}\end{cases}
$$

and similarly for the projection

$$
Q=\mathrm{s}-\lim _{t \rightarrow 0+} F^{t} .
$$

It is not difficult to construct the operator

$$
L_{1}^{-1}= \begin{cases}\sum_{k=1}^{\infty}\left(\lambda-\lambda_{k}\right)^{-1} e_{k} & \text { if } \lambda_{k} \neq \lambda \text { for all } k \in \mathbb{N}, \\ \sum_{k \in \mathbb{N}: k \neq \ell}\left(\lambda-\lambda_{k}\right)^{-1} e_{k} & \text { if } \lambda_{\ell}=\lambda \text { for some } \ell \in \mathbb{N} .\end{cases}
$$

By Theorem 10, for the Showalter-Sidorov problem (9) (11) we have

Corollary 4. Given $m, \lambda, \beta \in \mathbb{R}, \tau, q, \alpha \in \mathbb{R}_{+}, u_{0} \in \mathfrak{U}, f^{0} \in C^{1}\left((0, \tau) ; \mathfrak{F}^{0}\right)$, and $f^{1} \in C\left((0, \tau) ; \mathfrak{F}^{1}\right)$, there exists a unique solution $u \in C^{1}((0, \tau) ; \mathfrak{U})$ to problem $(9),(11)$; moreover, it is of the form

$$
u(t)=-M_{0}^{-1} f^{0}(t)+U^{t} u_{0}+\int_{0}^{t} U^{t-s} L_{1}^{-1} f^{1}(s) d s,
$$


here

$$
\begin{gathered}
\mathfrak{F}^{0}=\left\{\begin{array}{l}
\{0\}, \text { if } \lambda_{k} \neq \lambda \text { for all } k \in \mathbb{N} ; \\
\left\{f \in \mathfrak{F}: f_{k}=0, k \in \mathbb{N} \backslash\left\{\ell: \lambda_{\ell}=\lambda\right\}\right\} ;
\end{array}\right. \\
\mathfrak{F}^{1}=\left\{\begin{array}{l}
\mathfrak{F}, \text { if } \lambda_{k} \neq \lambda \text { for all } k \in \mathbb{N} ; \\
\left\{f \in \mathfrak{F}: f_{k}=0, \lambda_{k}=\lambda\right\} ;
\end{array}\right. \\
M_{0}^{-1}=\left\{\begin{array}{l}
\mathbb{O}, \text { if } \lambda_{k} \neq \lambda \text { for all } k \in \mathbb{N} ; \\
\sum_{k \in \mathbb{N}: \lambda_{k}=\lambda}\left(\alpha \lambda_{k}^{2}+\beta \lambda_{k}\right)^{-1} e_{k} .
\end{array}\right.
\end{gathered}
$$

\section{References}

1. Hille E., Phillips R.S. Functional Analysis and Semi-Groups. Providence, AMS, 1957.

2. Sviridyuk G.A., Fedorov V.E. [Semigroups of Operators with Kernels]. Bulletin of Chelyabinsk State University. Mathematics Mechanics, Informatics, 2002, no. 1, pp. 42-70. (in Russian)

3. Yosida K. Functional analysis. Berlin-Gottingen-Heidelberg, Springer-Verlag, 1965.

4. Sviridyuk G.A. [Sobolev Type Linear Equations and Strongly Continuous Semigroups of Resolving Operators with Kernels]. Dokl. Akad. Nauk USSR-Reports of the USSR Academy of Sciences, 1994, vol. 337, no. 5, pp. 581-584. (in Russian)

5. Sviridyuk G.A., Fedorov V.E. Linear Sobolev Type Equations and Degenerate Semigroups of Operators. Utrecht - Boston - Tokyo - Keln, VSP, 2003.

6. Fedorov V.E. [Strong Continuous Semigroups of Sobolev Type Equations in Locally Convex Spaces]. Neklassicheskie uravneniya matematicheskoy fiziki - Nonclassical Equation of Mathematical Physics. Novosibirsk, Publish in IM SB RAS, 2000, pp. 32-40. (in Russian)

7. Demidenko G.V., Uspenskii S.V. Partial Differential Equations and Systems not Solvable with Respect to the Highest-order Derivative. New York-Basel-Hong Kong, Marcel Dekker Inc., 2003.

8. Sveshnikov A.G., Al'shin A.B., Korpusov M.O., Pletner Yu.D. [Linear and Nonlinear Equation of Sobolev Type]. Moscow, FizMatLit Publ., 2007.

9. Zamyshlyaeva A.A. [Linear Sobolev Type Equations of Hihg Order]. Chelyabinsk, Publishing center of SUSU, 2012. (in Russian)

10. Manakova N.A. [Optimal Control Problem for Semilinear Sobolev Type Equations]. Chelyabinsk, Publishing center of SUSU, 2012. (in Russian)

11. Sagadeeva M.A. [Dichotomies of the Solutions for the Linear Sobolev Type Equations]. Chelyabinsk, Publishing center of SUSU, 2012. (in Russian) 
12. Sviridyuk G.A., Zagrebina S.A. Nonclassical Mathematical Physics Models. Bulletin of the South Ural State University. Series "Mathematical Modelling, Programming 8 Computer Software", 2012, no. 40(299), pp. 7-18. (in Russian)

13. Al-Delfi J.K. Quasi-Sobolev Spaces $\ell_{p}^{m}$. Bulletin of the South Ural State University. Series "Mathematics. Mechanics. Physics", 2013, vol. 5, no. 1, pp. 107-109. (in Russian)

14. Sviridyuk G.A. [Manifolds of Solutions of a Class of Evolution and Dynamic Equations]. Dokl. Akad. Nauk USSR - Reports of the USSR Academy of Sciences, 1989, vol. 304, no. 2, pp. 301-304. (in Russian)

15. Keller A.V., Al-Delfi J.K. Holomorphic Degenerate Groups of Operators in QuasiBanach Spaces. Bulletin of the South Ural State University. Series "Mathematics. Mechanics. Physics", 2015, vol. 7, no. 1, pp. 20-27. (in Russian)

16. Sviridyuk G.A., Manakova N.A. An Optimal Control of the Solutions of the InitialFinal Problem for Linear Sobolev Type Equations with Strongly Relatively p-Radial Operator. Semigroups of Operators - Theory and Applications. Series "Springer Proceedings in Mathematics \& Statistics", 2015, vol. 113, pp. 213-224.

17. Sviridyuk G.A., Zagrebina S.A. The Showalter - Sidorov Problem as Phenomena of the Sobolev-Type Equations. The Bulletin of Irkutsk State University. Series "Mathematics", 2010, vol. 3, no. 1, pp. 104-125. (in Russian)

18. Sagadeeva M.A., Shulepov A.N. The Approximations for Degenerate $C_{0}$-semigroup. Bulletin of the South Ural State University. Series "Mathematical Modelling, Programming \& Computer Software", 2013, vol. 6, no. 2, pp. 133-137. (in Russian)

Minzilya A. Sagadeeva, Candidate of Physico-Mathematical Sciences, Docent, Head of the Department of Mathematical Modeling, South Ural State University, Chelyabinsk, Russian Federation, sam79@74.ru.

Ammar Saheed Rashid, Master of Mathematics, Department of Equation of Mathematical Physics, South Ural State University, Chelyabinsk, Russian Federation,amargivara@gmail.com.

Received April 28, 2015 